PROCEEDINGS OF THE

AMERICAN MATHEMATICAL SOCIETY

Volume 126, Number 5, May 1998, Pages 1415-1423

S $0002-9939(98) 04227-0$

\title{
ON THE GROWTH OF POLYNOMIALS
}

\author{
D. DRYANOV AND Q. I. RAHMAN \\ (Communicated by Albert Baernstein II) \\ Dedicated to the memory of Professor Paul Erdös
}

\begin{abstract}
Let $f$ be a polynomial of degree $n$ having only real zeros and none in $(-1,1)$. We look for a sharp upper bound for $|f(z)|$ at an arbitrary point of the complex plane $\mathbb{C}$ in terms of the supremum norm on $[-1,1]$.
\end{abstract}

\section{INTRODUCTION}

Let $\mathcal{P}_{n}$ be the class of all polynomials of degree at most $n$. As usual, we denote by $T_{n}$ the $n$-th Chebyshev polynomial of the first kind. According to a classical result of P.L. Chebyshev (see [5] or [6]), if $f \in \mathcal{P}_{n}$ and $|f(x)| \leq 1$ for $-1 \leq x \leq 1$, then

$$
|f(x)| \leq\left|T_{n}(x)\right| \text { for } x \in \mathbb{R} \backslash[-1,1] .
$$

In (1) equality holds at some point $x_{0} \in \mathbb{R} \backslash[-1,1]$ if and only if $f(z) \equiv e^{i \gamma} T_{n}(z)$ for some real $\gamma$. It was noted by S. Bernstein [2] that if $f(z)$ is real for real $z$, then

$$
|f(z)| \leq\left|T_{n}(z)\right| \text { for }|z| \geq 1,
$$

even if $|f|$ is bounded by 1 only at the $n+1$ points $\cos \frac{\nu \pi}{n}, \nu=0,1, \ldots, n$, which are the extrema of $T_{n}$ in $[-1,1]$. Bernstein's paper went unnoticed, and his result was rediscovered by P. Erdös [4].

The polynomial $T_{n}$ is extremal for several other problems. For example, it was proved by A. Markov (see [5] or [6]) that if $f \in \mathcal{P}_{n}$ and $|f(x)| \leq 1$ for $-1 \leq x \leq 1$, then

$$
\max _{-1 \leq x \leq 1}\left|f^{\prime}(x)\right| \leq n^{2}=\max _{-1 \leq x \leq 1}\left|T^{\prime}{ }_{n}(x)\right| .
$$

All the zeros of $T_{n}$ are real and lie in the open interval $(-1,1)$. This suggests that the above inequalities can be considerably improved if the zeros of $f$ are all real but none of them lies in $(-1,1)$. As regards (3), P. Erdös [3]; see in particular the second half of p. 311 proved the following result.

Theorem A. Let $f \in \mathcal{P}_{n}$ be such that $|f(x)| \leq 1$ for $-1 \leq x \leq 1$. If the zeros of $f$ are all real and lie on $\mathbb{R} \backslash(-1,1)$, then

$$
\max _{-1 \leq x \leq 1}\left|f^{\prime}(x)\right| \leq \frac{1}{2}\left(1-\frac{1}{n}\right)^{-n+1} n .
$$

Received by the editors October 16, 1996.

1991 Mathematics Subject Classification. Primary 30A10, 30C10, 30D15, 41A17.

(C)1998 American Mathematical Society 
The inequality becomes an equality for polynomials of the form

$$
\frac{e^{i \gamma} n^{n}(1+x)^{n-1}(1-x)}{2^{n}(n-1)^{n-1}}, \frac{e^{i \gamma} n^{n}(1-x)^{n-1}(1+x)}{2^{n}(n-1)^{n-1}}, \text { where } \gamma \in \mathbb{R} .
$$

Here we obtain a result which may be seen as an analogue of (2) for polynomials with zeros restricted as in Theorem A.

For each positive integer $n$ and $k=0,1, \ldots, n$ let $\eta_{n, k}:=-1+\frac{2 k}{n}$. Denote by $\mathcal{P}_{n, \mathbb{R}, 1}$ the family of all polynomials $f$ in $\mathcal{P}_{n}$ which have only real zeros, none of which lies in $(-1,1)$, and satisfy $|f(x)| \leq 1$ for all $x \in F_{n}:=\left\{\eta_{n, k}: 0 \leq k \leq n\right\}$. Furthermore, for $k=0,1, \ldots, n$, let

$$
q_{n, k}(z):=\frac{n^{n}}{2^{n} k^{k}(n-k)^{n-k}}(1+z)^{k}(1-z)^{n-k} .
$$

Note that $\max _{-1 \leq x \leq 1}\left|q_{n, k}(x)\right|=q_{n, k}\left(\eta_{n, k}\right)=1$ for each $k$. We prove

Theorem 1. Let $\Omega$ be the union of the open disks

$$
\left\{z \in \mathbb{C}:\left|z \pm \frac{i}{\sqrt{3}}\right|<\frac{2}{\sqrt{3}}\right\}
$$

and $\mathbb{S}:=\mathbb{C} \backslash \Omega$. If $f \in \mathcal{P}_{n, \mathbb{R}, 1}$, then for all $z \in \mathbb{S}$

$$
|f(z)| \leq \max _{0 \leq k \leq n}\left|q_{n, k}(z)\right| .
$$

Remark 1. Our proof of the theorem will show that except when $z= \pm i \sqrt{3}$ and $n=1$, strict inequality holds in (5) unless $f(z) \equiv e^{i \gamma} q_{n, k}(z)$ for some $k$ and $\gamma \in \mathbb{R}$. If $n=1$, then $|f( \pm i \sqrt{3})|$ is also maximized by each constant of modulus 1 .

\section{Proof of Theorem 1}

There is nothing to prove for $z= \pm 1$. Besides, for reasons of symmetry, it is enough to prove (5) for all

$$
z \in \mathbb{E}:=\mathbb{S} \cap\{x+i y: x \geq 0\} \backslash\{1\} .
$$

The result is obtained in several steps.

Step 1. Given any point $\zeta \in \mathbb{E}$, let $\sigma_{\zeta}:=\sup _{f \in \mathcal{P}_{n, \mathbb{R}, 1}}|f(\zeta)|$. There exists a polynomial $g \in \mathcal{P}_{n, \mathbb{R}, 1}$ such that $|g(\zeta)|=\sigma_{\zeta}$. For this we observe that if

$$
\psi(z):=\prod_{k=0}^{n}\left(z-\eta_{n, k}\right),
$$

then for all $z \in \mathbb{C}$ we have

$$
f(z)=\sum_{k=0}^{n} f\left(\eta_{n, k}\right) \frac{\psi(z)}{\psi^{\prime}\left(\eta_{n, k}\right)\left(z-\eta_{n, k}\right)} .
$$

From this it follows that the polynomials in $\mathcal{P}_{n, \mathbb{R}, 1}$ are uniformly bounded on every compact subset of $\mathbb{C}$, and so they form a normal family [1, p.216]. Consequently, there exists a polynomial $g \in \mathcal{P}_{n}$ such that $\left|g\left(\eta_{n, k}\right)\right| \leq 1$ for $k=0,1, \ldots, n$ and $|g(\zeta)|=\sigma_{\zeta}$. Obviously, $g$ cannot be identically zero. So by a well-known theorem of Hurwitz [1, p.176] it must belong to $\mathcal{P}_{n, \mathbb{R}, 1}$. We call such a polynomial extremal. 
Step 2. Let

$$
\mathcal{E}_{\zeta}:=\left\{g \in \mathcal{P}_{n, \mathbb{R}, 1}:|g(\zeta)|=\sigma_{\zeta}\right\} .
$$

It is clear that if $g \in \mathcal{E}_{\zeta}$, then $\max _{1 \leq k \leq n}\left|g\left(\eta_{n, k}\right)\right|$ must be equal to 1 . Besides, it must be of degree $n$ unless $n=1$ and $\zeta=i \sqrt{3}$. Indeed, if a polynomial $g \in \mathcal{P}_{n, \mathbb{R}, 1}$ is of degree $n-j<n$, then

$$
g_{+}(z):=\left(\frac{1+z}{2}\right)^{j} g(z)
$$

also belongs to $\mathcal{P}_{n, \mathbb{R}, 1}$, and $\left|g_{+}(\zeta)\right|>|g(\zeta)|$ if $|\zeta+1|>2$. In particular, $\left|g_{+}(\zeta)\right|>$ $|g(\zeta)|$ for all $\zeta \in \mathbb{E}, \zeta \neq i \sqrt{3}$, and so $g$ cannot be extremal for any $\zeta \in \mathbb{E}$ except possibly for $\zeta=i \sqrt{3}$. Now let $n \geq 2$ and suppose that a polynomial $g \in \mathcal{P}_{n, \mathbb{R}, 1}$ of degree $n-j<n$ is extremal for $\zeta=i \sqrt{3}$. Without loss of generality we may assume $g$ to be positive on $(-1,1)$. Since $f(z):=1-z^{2}$ belongs to $\mathcal{P}_{n, \mathbb{R}, 1}$ for $n \geq 2$ and $|f(i \sqrt{3})|=4>1$, it follows that $g(z)$ cannot be identically equal to 1 , i.e. $g$ cannot be of degree 0 . Next we note that $g$ cannot attain its maximum on the set $\left\{\eta_{n, k}: k=0,1, \ldots, n\right\}$ at both -1 and +1 . If it did, it would have at least three critical points between the largest zero in $(-\infty,-1]$ and the smallest zero in $[1, \infty)$. But that is not possible since the zeros of $g$ are all real. Hence if $g_{-}(z):=$ $((1-z) / 2)^{j} g(z)$, then either $\max _{0 \leq k \leq n}\left|g_{+}\left(\eta_{n, k}\right)\right|<1$ or $\max _{0 \leq k \leq n}\left|g_{-}\left(\eta_{n, k}\right)\right|<1$. Since $\left|g_{+}(i \sqrt{3})\right|=\left|g_{-}(i \sqrt{3})\right|=|g(i \sqrt{3})|$, we see that $g \notin \mathcal{E}_{i \sqrt{3}}$.

Step 3. In order to prove the theorem we need to show that if $g \in \mathcal{E}_{\zeta}$ and $g(\alpha)=0$, then $\alpha$ is either +1 or -1 . First of all we show that $\alpha$ cannot lie in $(-\infty,-1)$.

Let us agree to denote by $A, B, P$ and $X$ the points of the complex plane which represent the numbers $-1,+1, \zeta$ and $\alpha$, respectively. Assume that $\alpha \in(-\infty,-1)$. It is easily seen that in this situation

$$
\left|\frac{1-\alpha}{2} \frac{z+1}{z-\alpha}\right| \leq 1 \quad \text { for } z \in[-1,1]
$$

Furthermore,

$$
\left|\frac{1-\alpha}{2} \frac{\zeta+1}{\zeta-\alpha}\right|=\left|\frac{1-\alpha}{\zeta-\alpha}\right|\left|\frac{\zeta+1}{2}\right|=\frac{\sin \widehat{X P B}}{\sin \widehat{X B P}} \frac{\sin \widehat{A B P}}{\sin \widehat{A P B}}=\frac{\sin \widehat{X P B}}{\sin \widehat{A P B}}>1 .
$$

Hence, the polynomial

$$
g_{1}(z):=\frac{1-\alpha}{2} \frac{z+1}{z-\alpha} g(z)
$$

belongs to $\mathcal{P}_{n, \mathbb{R}, 1}$, but $\left|g_{1}(\zeta)\right|>|g(\zeta)|$, contradicting the assumption that $g \in \mathcal{E}_{\zeta}$.

It remains to show that if $g \in \mathcal{E}_{\zeta}$ and $g(\alpha)=0$, then $\alpha$ cannot lie in $(1, \infty)$.

Step 4. For each $\varphi \in\left(0, \frac{\pi}{2}\right)$, we denote by $\mathcal{L}_{\varphi}$ the line

$$
z=-1+t e^{i \varphi} \quad(-\infty<t<\infty)
$$

and by $\mathbb{H}_{\varphi}$ that half-plane bounded by $\mathcal{L}_{\varphi}$ which contains the infinite interval $[-1, \infty)$. Let

$$
t_{1}=t_{1}(\varphi):=\inf \left\{t:-1+t e^{i \varphi} \in \mathbb{E}\right\} \quad\left(0<\varphi<\frac{\pi}{2}\right)
$$

and consider the half-lines

$$
\mathcal{L}_{\varphi}^{+}:-1+t e^{i \varphi}\left(t_{1} \leq t<\infty\right) .
$$


Take any point $P$ on $\mathcal{L}_{\varphi}^{+}$, i.e. $P$ represents $\zeta=\zeta(t)=-1+t e^{i \varphi}$, where $t \geq t_{1}$. Take another point $Q$ on the same half-line such that $P$ is an interior point of the line segment $A Q$.

Before we go on, we need to take a point $C$ on the positive real axis and draw three half-lines $\Lambda_{1}, \Lambda_{2}, \Lambda_{3}$ contained in $\mathbb{H}_{\varphi}$ with $P$ as initial point. The half-lines are drawn so that $\Lambda_{1}$ makes with $\overrightarrow{P B}$ an angle equal to $\widehat{A P B}, \Lambda_{2}$ makes with $\overrightarrow{P B}$ an angle equal to $\widehat{P B C}$, and $\Lambda_{3}$ makes with $\overrightarrow{P Q}$ an angle equal to $\widehat{A P B}$.

At this stage the reader will find it useful to note that the chord $A B$ subtends an angle $\frac{\pi}{3}$ at each point of the circle $\left|z-\frac{i}{\sqrt{3}}\right|=\frac{2}{\sqrt{3}}$ which lies in the upper half-plane. It should be clear that except when $\zeta=i \sqrt{3}$, the half-line $\Lambda_{1}$ intersects the positive real axis. Denote the point of intersection by $L(\zeta)$, or by $L$ for brevity. We may say that $L(i \sqrt{3})=\infty$. Let $a=a(\zeta)$ be the distance of $L$ from the origin. Thus $a(\zeta)<+\infty$ unless $\zeta=i \sqrt{3}$.

The half-line $\Lambda_{2}$ intersects the positive real axis if and only if $\widehat{A P B}+\widehat{P B C}<$ $\pi-\widehat{P A B}$, which is equivalent to

$$
\widehat{A P B}+\widehat{P A B}<\frac{\pi}{2}
$$

since $\widehat{P B C}=\widehat{P A B}+\widehat{A P B}$. Thus $\Lambda_{2}$ intersects the positive real axis if and only if $\zeta \in \mathbb{E} \cap\{x+i y: x>1, y>0\}$. Denote the point of intersection by $M(\zeta)$, or by $M$ if there is no ambiguity. We say that $M$ is the point at infinity for all $\zeta \in \mathbb{E} \backslash\{x+i y: x>1, y>0\}$. Let $b=b(\zeta)$ be the distance of $M$ from the origin. Note that $b\left(-1+t e^{i \varphi}\right)<+\infty$ for each $t \geq t_{1}$ when $0<\varphi<\frac{\pi}{6}$, and only for $t>2 \sec \varphi$ when $\varphi \in\left[\frac{\pi}{6}, \frac{\pi}{2}\right)$.

The half-line $\Lambda_{3}$ intersects the positive real axis if and only if $\widehat{A P B}>\widehat{P A B}$. This cannot be the case if $P$ lies on $\mathcal{L}_{\varphi}^{+}$for any $\varphi \in\left[\frac{\pi}{3}, \frac{\pi}{2}\right)$. If $\varphi \in\left(0, \frac{\pi}{3}\right)$ and $\zeta=-1+t e^{i \varphi}$, then $\widehat{A P B}>\widehat{P A B}$ if and only if $t_{1}(\varphi)=\frac{2}{\sqrt{3}} \sin \varphi+2 \cos \varphi \leq t<$ $4 \cos \varphi$. If $\Lambda_{3}$ intersects the positive real axis, we denote the point of intersection by $N(\zeta)$ or simply by $N$. Let $c=c(\zeta)$ be the distance of $N$ from the origin.

Step 5. We need to compare the quantities $a(\zeta), b(\zeta)$ and $c(\zeta)$. Note that $c(\zeta)=$ $+\infty$ if $\zeta \in \mathcal{L}_{\varphi}^{+}$, where $\frac{\pi}{3} \leq \varphi<\frac{\pi}{2}$, and also when $\zeta=\zeta(t)=-1+t e^{i \varphi}$, where $\varphi \in\left(0, \frac{\pi}{3}\right)$ but $t \geq 4 \cos \varphi$. For each $\varphi \in\left(0, \frac{\pi}{3}\right)$ the quantity $c\left(-1+t e^{i \varphi}\right)$ increases as $t$ increases from $t_{1}(\varphi)$ to $4 \cos \varphi$.

It follows that

$$
b(\zeta) \leq c(\zeta)
$$

except possibly when $\zeta$ is of the form $-1+t e^{i \varphi}$, where $0<\varphi<\frac{\pi}{3}$ and $t_{1} \leq t<$ $4 \cos \varphi$.

Since $b(\zeta)=+\infty$ for $\zeta \in \mathbb{E} \backslash\{x+i y: x>1, y>0\}$, we trivially have

$$
c(\zeta)<b(\zeta)
$$

if $\zeta=-1+t e^{i \varphi}$, where $\frac{\pi}{4} \leq \varphi<\frac{\pi}{3}$ and $t_{1}(\varphi) \leq t<4 \cos \varphi$. At least one of $c(\zeta)$ and $b(\zeta)$ will be $+\infty$ for $\varphi \in\left[\frac{\pi}{4}, \frac{\pi}{3}\right)$, because $2 \sec \varphi \geq 4 \cos \varphi$ for such values of $\varphi$.

For each $\varphi \in\left(0, \frac{\pi}{4}\right)$ there exists a number

$$
t_{2}(\varphi) \in\left(\max \left\{t_{1}(\varphi), 2 \sec \varphi\right\}, 4 \cos \varphi\right)
$$


such that

$$
c\left(-1+t_{2}(\varphi) e^{i \varphi}\right)=b\left(-1+t_{2}(\varphi) e^{i \varphi}\right)
$$

whereas $c\left(-1+t e^{i \varphi}\right)<b\left(-1+t e^{i \varphi}\right)$ or $c\left(-1+t e^{i \varphi}\right)>b\left(-1+t e^{i \varphi}\right)$ according as $t_{1}(\varphi) \leq t<t_{2}(\varphi)$ or $t>t_{2}(\varphi)$, respectively. Indeed, for $\zeta=-1+t e^{i \varphi} \in$ $\mathcal{L}_{\varphi}^{+}$the angles $\widehat{A P B}, \widehat{P B C}$ decrease as $t$ increases; so there is one and only one $\zeta \in \mathcal{L}_{\varphi}^{+}$corresponding to which $\widehat{A P B}+\widehat{P B C}=\pi-\widehat{A P B}$, and this occurs for $\frac{\pi}{3}<\widehat{P B C}<\frac{\pi}{2}$. For such $\zeta$ the point $M(\zeta)$ coincides with $N(\zeta) ; N(\zeta)$ lies to the left of $M(\zeta)$ or to the right of $M(\zeta)$ according as $\widehat{A P B}+\widehat{P B C}>\pi-\widehat{A P B}$ or $\widehat{A P B}+\widehat{P B C}<\pi-\widehat{A P B}$, respectively.

Since $\widehat{P B C}=\widehat{A P B}+\widehat{P A B}>\widehat{A P B}$, it follows that $a(\zeta)<b(\zeta)$ for all $\zeta \in$ $\mathbb{E} \backslash\{i \sqrt{3}\}$, whereas $a(i \sqrt{3})=b(i \sqrt{3})=+\infty$. If $\zeta=-1+t e^{i \varphi}$, where $0<\varphi<\frac{\pi}{2}$ and $t>t_{1}(\varphi)$, then $\widehat{A P B}<\frac{\pi}{3}$; so $\widehat{A P L}<\frac{2 \pi}{3}$, whereas $\widehat{A P N}>\frac{2 \pi}{3}$, which implies that $a(\zeta)<c(\zeta)$. The same can be said if $t=t_{1}(\varphi)$ and $\varphi \in\left(\frac{\pi}{3}, \frac{\pi}{2}\right)$. However, if $t=t_{1}(\varphi)$ and $\varphi \in\left(0, \frac{\pi}{3}\right)$, then $a(\zeta)=c(\zeta)<+\infty$, and for $\varphi=\frac{\pi}{3}$ we have $a(i \sqrt{3})=c(i \sqrt{3})=+\infty$.

The above observations about $a(\zeta), b(\zeta)$ and $c(\zeta)$ allow us to conclude that the interval $(1,+\infty)$ can be the same as $(1, c(\zeta))$. But in the case $c(\zeta)<+\infty$, we can express $(1,+\infty)$ as $(1, c(\zeta)] \cup[a(\zeta), b(\zeta)) \cup[b(\zeta),+\infty)$ if $b(\zeta)<+\infty$ and as $(1, c(\zeta)] \cup[a(\zeta), b(\zeta))$ if $b(\zeta)=+\infty$.

Step 6. We are finally ready to prove that if $g \in \mathcal{E}_{\zeta}$ and $g(\alpha)=0$, then $\alpha$ cannot lie in $(1,+\infty)$.

I. Let $\alpha \in(1, c]$ if $c<+\infty$; otherwise let $\alpha \in(1,+\infty)$. It is clear that

$$
\left|\frac{z-1}{z-\alpha} \frac{\alpha+1}{2}\right| \leq 1 \quad \text { for } z \in[-1,1] .
$$

Since

$$
\left|\frac{\zeta-1}{2} \frac{\alpha+1}{\zeta-\alpha}\right|=\frac{\sin \widehat{P A B}}{\sin \widehat{A P B}} \frac{\sin \widehat{A P X}}{\sin \widehat{P A X}}=\frac{\sin \widehat{A P X}}{\sin \widehat{A P B}},
$$

and

$$
\widehat{A P B}<\widehat{A P X} \leq \widehat{A P N}=\pi-\widehat{A P B}
$$

it follows that $\sin \widehat{A P B} \leq \sin \widehat{A P X}$, i.e. $\left|\frac{\zeta-1}{\zeta-\alpha} \frac{\alpha+1}{2}\right| \geq 1$, where the inequalities are strict unless $X$ coincides with $N(\zeta)$. Hence the polynomial

$$
g_{2}(z):=\frac{z-1}{z-\alpha} \frac{\alpha+1}{2} g(z)
$$

belongs to $\mathcal{P}_{n, \mathbb{R}, 1}$ and $\left|g_{2}(\zeta)\right| \geq|g(\zeta)|$, where the inequality is strict unless $c(\zeta)<$ $+\infty$ and $\alpha=c(\zeta)$. So the assumption $g \in \mathcal{E}_{\zeta}$ is contradicted except in such a situation. Since $c(i \sqrt{3})=+\infty$, we may hereafter assume $\zeta \neq i \sqrt{3}$.

II. Now let $\alpha \in[a, b)$. There exists a point $X_{1}$ in $(-\infty,-1)$ such that $\widehat{X_{1} P B}=$ $\widehat{B P X}$. Denote by $-\alpha_{1}$ the corresponding real number. The function

$$
g_{3}(z):=\frac{z+\alpha_{1}}{z-\alpha} \frac{\alpha-1}{\alpha_{1}+1} g(z)
$$


is easily seen to belong to $\mathcal{P}_{n, \mathbb{R}, 1}$. Besides,

$$
\left|\frac{\zeta+\alpha_{1}}{1+\alpha_{1}}\right|\left|\frac{\alpha-1}{\zeta-\alpha}\right|=\frac{\sin \widehat{X_{1} B P}}{\sin \widehat{X_{1} P B}} \frac{\sin \widehat{B P X}}{\sin \widehat{P B X}},
$$

i.e. $\left|g_{3}(\zeta)\right|=|g(\zeta)|$. Thus the function

$$
h_{3}(z):=\frac{z+1}{2} \frac{1+\alpha_{1}}{z+\alpha_{1}} g_{3}(z)
$$

belongs to $\mathcal{P}_{n, \mathbb{R}, 1}$ and

$$
\left|h_{3}(z)\right|=\left|\frac{\zeta+1}{2}\right|\left|\frac{1+\alpha_{1}}{\zeta+\alpha_{1}}\right||g(\zeta)|=\frac{\sin \widehat{A B P}}{\sin \widehat{A P B}} \frac{\sin \widehat{X_{1} P B}}{\sin \widehat{X_{1} B P}}|g(\zeta)| \geq|g(\zeta)|,
$$

where the inequality is strict unless $\alpha=a(\zeta)$. So, we get a contradiction except when $\alpha=a(\zeta)$.

III. Next, let $b<+\infty$ and $\alpha \geq b$. The polynomial

$$
g_{4}(z):=\frac{\alpha-1}{z-\alpha} g(z)
$$

belongs to $\mathcal{P}_{n-1, \mathbb{R}, 1}$, and

$$
\left|g_{4}(\zeta)\right|=\frac{\sin \widehat{B P X}}{\sin \widehat{P B X}}|g(\zeta)| \geq|g(\zeta)|
$$

where the inequality is strict unless $\alpha=b(\zeta)$. But there is really no extremal polynomial of degree less than $n$, since $\zeta \neq i \sqrt{3}$. This is a contradiction.

Summarizing the above conclusions, we see that we have got a contradiction except when $c(\zeta)<+\infty$ and $\alpha=c(\zeta)$ or when $\alpha=a(\zeta)$. Comparing parts I, II and III, we see that $\alpha=c(\zeta)$ and $\alpha=a(\zeta)$ are covered if $a(\zeta)<c(\zeta)$. All that remains is the case $\alpha=a(\zeta)=c(\zeta)<+\infty$. But this occurs only when $\zeta=\zeta_{1}:=-1+t_{1} e^{i \varphi}$, where $0<\varphi<\frac{\pi}{3}$, i.e. $\zeta$ lies on the circle $\left|z-\frac{i}{\sqrt{3}}\right|=\frac{2}{\sqrt{3}}$. In that case we introduce the polynomial

$$
g_{5}(z):=\frac{a(\zeta)+1}{z-a(\zeta)} \frac{z-1}{2} g(z) .
$$

First we note that $g$ must vanish at -1 . If not, the polynomial $g(-z)$ would have all its zeros in $(-\infty,-1]$, and $|g(-\zeta)|$ would be larger than $|g(\zeta)|$, contrary to the assumption that $g \in \mathcal{E}_{\zeta}$. Next we note that $\frac{a(\zeta)+1}{x-a(\zeta)} \frac{x-1}{2}$ decreases from 1 to 0 as $x$ increases from -1 to +1 . This means that $\max _{x \in F_{n}}|g(x)|$ and $\max _{x \in F_{n}}\left|\frac{a(\zeta)+1}{x-a(\zeta)} \frac{x-1}{2}\right|$ are not attained at the same point of $F_{n}$. Hence

$$
\max _{x \in F_{n}}\left|g_{5}(x)\right|=\mu \max _{x \in F_{n}}|g(x)|,
$$

where $\mu \in(0,1)$. We get a contradiction with the fact that if $g_{5} \in \mathcal{E}_{\zeta}$ then $\max \left\{\left|g_{5}\left(\eta_{n, k}\right)\right|: k=0,1, \ldots, n\right\}$ must be equal to 1 .

It remains to consider points $\zeta \in(1,+\infty)$. Clearly $g(\zeta)$ cannot be zero. So we have to consider two possibilities, namely, $\alpha \in(\zeta,+\infty)$ and $\alpha \in(1, \zeta)$. In the first case, we may consider $g_{4}(z):=\frac{\alpha-1}{\alpha-z} g(z)$ to see that $g$ cannot belong to $\mathcal{E}_{\zeta}$ if $g(\alpha)=0$. In the second case, $g_{2}(z)=\frac{1-z}{\alpha-z} \frac{\alpha+1}{2} g(z)$ shows the same. 


\section{Determination of $\max _{0 \leq k \leq n}\left|q_{n, k}(z)\right|$ FOR a Given $z$}

First let $\operatorname{Re}(z) \geq 0$, i.e. $\left|\frac{1+z}{1-z}\right| \geq 1$. Then, for $\left[\frac{n+1}{2}\right] \leq k \leq n$, we have $\left|q_{n, k}(z) / q_{n, n-k}(z)\right| \geq 1$. Hence

$$
\Theta(z):=\max _{0 \leq k \leq n}\left|q_{n, k}(z)\right|=\max _{\left[\frac{n+1}{2}\right] \leq k \leq n}\left|q_{n, k}(z)\right| .
$$

For $0 \leq t \leq n$, let

$$
\rho(t):=\frac{(t+1)^{t+1}(n-t-1)^{n-t-1}}{t^{t}(n-t)^{n-t}},
$$

where, as usual, $0^{0}=1$. Examining its logaritmic derivative, we see that $\rho(t)$ is strictly increasing on $[0, n-1]$. Hence

$$
\rho\left(\left[\frac{n-1}{2}\right]\right)<\rho\left(\left[\frac{n+1}{2}\right]\right)<\cdots<\rho(n-1) .
$$

Furthermore, it is easily checked that

$$
\rho\left(\left[\frac{n-1}{2}\right]\right)<1<\rho\left(\left[\frac{n+1}{2}\right]\right) \text { if } n \text { is even, }
$$

whereas

$$
\rho\left(\left[\frac{n-1}{2}\right]\right)=1<\rho\left(\left[\frac{n+1}{2}\right]\right) \text { if } n \text { is odd . }
$$

It is clear that if $z \neq 1$ and $w(z):=\frac{1+z}{1-z}$, then

$$
\left|q_{n, k+1}(z) / q_{n, k}(z)\right| \geq 1 \text { for } 0 \leq k \leq n-1
$$

if and only if

$$
|w(z)|=\left|\frac{1+z}{1-z}\right| \geq \rho(k),
$$

where equality holds in (10) if and only if it does in (11). Hence for $\left[\frac{n-1}{2}\right] \leq k \leq$ $n-1$,

$$
\left|q_{n, k+1}(z)\right| \geq\left|q_{n, k}(z)\right| \text { if and only if } \rho(k) \leq|w(z)|<+\infty .
$$

Besides, $\left|q_{n, k+1}(z)\right|=\left|q_{n, k}(z)\right|$ only if $|w(z)|=\rho(k)$. Because of (7), it follows that for each integer $j$ such that $n \geq j \geq\left[\frac{n-1}{2}\right]$, we have

$$
\left|q_{n, j}(z)\right| \geq\left|q_{n, k}(z)\right| \text { for all } k \leq j-1 \text { if } \rho(j-1) \leq|w(z)| \text {, }
$$

where $\left|q_{n, j}(z)\right|=\left|q_{n, k}(z)\right|$ only if $k=j-1$ and $|w(z)|=\rho(j-1)$. In addition, for $n>j \geq\left[\frac{n-1}{2}\right]$,

$$
\left|q_{n, j}(z)\right| \geq\left|q_{n, k}(z)\right| \quad \text { for } j<k \leq n \quad \text { if } \quad|w(z)| \leq \rho(j),
$$

where $\left|q_{n, j}(z)\right|=\left|q_{n, k}(z)\right|$ only if $k=j+1$ and $|w(z)|=\rho(j)$. Thus, setting $\rho(n)=+\infty$, we see that for each integer $j$ such that $n \geq j \geq\left[\frac{n+1}{2}\right]$,

$$
\max _{0 \leq k \leq n, k \neq j}\left|q_{n, k}(z)\right|<\left|q_{n, j}(z)\right| \quad \text { if } \rho(j-1)<|w(z)|<\rho(j) .
$$

It may be added that if $|w(z)|=\rho(j-1)$, then $\left|q_{n, j}(z)\right|=\left|q_{n, j-1}(z)\right|$. Thus, for any given $z$ belonging to the closed right half-plane, $\Theta(z)$ is attained by $\left|q_{n, j}(z)\right|$ alone if $\rho(j-1)<|w(z)|<\rho(j)$; it is also attained by $\left|q_{n, j-1}(z)\right|$ if $|w(z)|=\rho(j-1)$. Each point $z$ belonging to the closed right half-plane $\mathbb{H}^{+}$is covered, since for each such $z$ there exists, in view of (8) and (9), an integer $j$ in $\left[\left[\frac{n+1}{2}\right], n\right]$ such that 
$\rho(j-1) \leq|w(z)|=\left|\frac{1+z}{1-z}\right|<\rho(j)$. To determine $\Theta(z)$ for points belonging to the left half-plane, it suffices to observe that $\Theta(z)=\Theta(-z)$ for all $z$.

For $\left[\frac{n+1}{2}\right] \leq k \leq n-1$ let

$$
c_{k}:=\frac{(\rho(k))^{2}+1}{(\rho(k))^{2}-1}, r_{k}:=\frac{2 \rho(k)}{(\rho(k))^{2}-1} .
$$

It is easily checked that $c_{k}-r_{k}$ increases with $k$, whereas $c_{k}+r_{k}$ decreases. So if $\mathbb{D}_{k}:=\left\{z \in \mathbb{C}:\left|z-c_{k}\right|<r_{k}\right\}$, then

$$
\mathbb{D}_{l} \supset \overline{\mathbb{D}}_{m} \quad \text { if } \quad\left[\frac{n+1}{2}\right] \leq l<m \leq n-1 .
$$

Now note that $|w(z)| \geq \rho(k)$ for some integer $k$ such that $\left[\frac{n+1}{2}\right] \leq k \leq n-1$ if and only if $z \in \overline{\mathbb{D}}_{k}$. We therefore have the following:

Theorem 2. Let $\Theta(z), \mathbb{H}^{+}$and $\Omega_{k}$ be as above. If $z \in \overline{\mathbb{D}}_{n-1}$, then $\Theta(z)=$ $\left|q_{n, n}(z)\right|$; if $z \in \overline{\mathbb{D}}_{n-2} \backslash \mathbb{D}_{n-1}$, then $\Theta(z)=\left|q_{n, n-1}(z)\right|$. More generally, if $z \in$ $\overline{\mathbb{D}}_{k} \backslash \mathbb{D}_{k+1}$ for some $k$ such that $\left[\frac{n+1}{2}\right] \leq k \leq n-2$, then $\Theta(z)=\left|q_{n, k+1}(z)\right|$. If $z \in \mathbb{H}^{+} \backslash \mathbb{D}_{\left[\frac{n+1}{2}\right]}$, then $\Theta(z)=\left|q_{n,\left[\frac{n+1}{2}\right]}(z)\right|$.

Remark 2. It may be noted that if $\left|z-c_{k}\right|=r_{k}$ for some $k$ such that $\left[\frac{n+1}{2}\right] \leq$ $k \leq n-1$, then $\Theta(z)=\left|q_{n, k+1}(z)\right|=\left|q_{n, k}(z)\right|$. Furthermore, if $n$ is odd and $\operatorname{Re}(z)=0$, then $\Theta(z)=\left|q_{n,\left[\frac{n+1}{2}\right]}(z)\right|=\left|q_{n,\left[\frac{n-1}{2}\right]}(z)\right|$.

\section{Some Additional REMARKS}

Remark 3. Even if we assume $|f(x)|$ to be bounded by 1 for all $x \in[-1,1]$, it is clearly not possible to improve upon (5). But for (5) to remain true for all $z \in \mathbb{S}$, do we have to assume that $\left|f\left(\eta_{n, k}\right)\right| \leq 1$ for $0 \leq k \leq n$ ? The answer is yes. In fact, if we require $|f(x)|$ to be bounded above by 1 on any closed subset $F$ of $[-1,1]$ which does not contain one of the above $n+1$ points, say $\eta_{n, j}$, then (5) will fail at least for all $z \in \mathbb{C}$, where $\Theta(z)=\left|q_{n, j}(z)\right|$. This is because there exists $\delta>0$ such that $\left|(1+\delta) q_{n, j}(x)\right| \leq 1$ for all $x \in F$.

Remark 4 . What can we say about $|f(z)|$ when $z \notin \mathbb{S}$, i.e. $z \in \Omega$ ? First of all we wish to point out that $\mathbb{S}$ is independent of $n$. The answer to the question depends on $n$. Note that inequality (5) does not hold for any $z$ belonging to the intersection $\Delta$ of the two disks $|1+z|<2$ and $|1-z|<2$ in case $n=1$. This follows from the definition of $\Delta$. Now consider the polynomial $f(z):=\frac{1}{2}(1+z)(2-z)$, which satisfies the conditions of Theorem 1 in case $n=2$. Comparing $|f(1+i y)|$ with $\left|q_{2,0}(1+i y)\right|,\left|q_{2,1}(1+i y)\right|$ and $\left|q_{2,2}(1+i y)\right|$, we see that

$$
|f(z)|>\max _{0 \leq k \leq 2}\left|q_{2, k}(z)\right|
$$

if $z=1+i y, 0<|y|<\frac{1}{\sqrt{3}}$. Note that these points lie in $\Omega \backslash \Delta$. On the other hand, not only for $n=2$ but for all even $n$, we have $|f(i y)|=|f(0)| \prod_{\nu=1}^{n}\left|1-i y x_{\nu}\right|$, where $-1 \leq x_{\nu} \leq 1$ for $\nu=1, \ldots, n$. Hence,

$$
|f(i y)| \leq|f(0)|\left(1+y^{2}\right)^{\frac{n}{2}}=\left|q_{n, \frac{n}{2}}(i y)\right|,
$$

i.e. (5) holds at all points of the imaginary axis in case $n$ is even. 
Remark 5. Although a polynomial $f$ of degree $n$ having only real zeros none of which lies in $(-1,1)$ is not completely determined by the value it takes at any point $\alpha$ in $(-1,1)$, its modulus at any point $z \in \mathbb{C}$ can be estimated in terms of $n$ and $|f(\alpha)|$. To see this note that $f(z)=f(0) \prod_{\nu=1}^{n}\left(1-z x_{\nu}\right)$, where $-1 \leq x_{\nu} \leq 1$ for $\nu=1, \ldots, n$. For all $z$,

$$
\left|1-z x_{\nu}\right| \leq \max \{|1+z|,|1-z|\} .
$$

Hence, if $|f(0)| \leq 1$, then

$$
|f(z)| \leq|f(0)| \max \left\{|1+z|^{n},|1-z|^{n}\right\}=\left\{\begin{array}{lll}
|1+z|^{n} & \text { if } \operatorname{Re}(z) \geq 0 \\
|1-z|^{n} & \text { if } \operatorname{Re}(z) \leq 0 .
\end{array}\right.
$$

If $|f(\alpha)| \leq 1$, where $\alpha \in(-1,0) \cup(0,1)$, then

$$
F(w):=(\alpha w+1)^{n} f\left(\frac{w+\alpha}{\alpha w+1}\right)
$$

is a polynomial of degree at most $n$ having only real zeros none of which lies in $(-1,1)$. Furthermore, $|F(0)|=|f(\alpha)| \leq 1$, and so by (12)

$$
|\alpha w+1|^{n} f\left(\frac{w+\alpha}{\alpha w+1}\right)=|F(w)| \leq \max \left\{|1+w|^{n},|1-w|^{n}\right\} .
$$

Replacing $\frac{w+\alpha}{\alpha w+1}$ by $z$, we easily conclude that if $|f(\alpha)| \leq 1$, where $\alpha \in(-1,0) \cup$ $(0,1)$, then for all $z \in \mathbb{C}$,

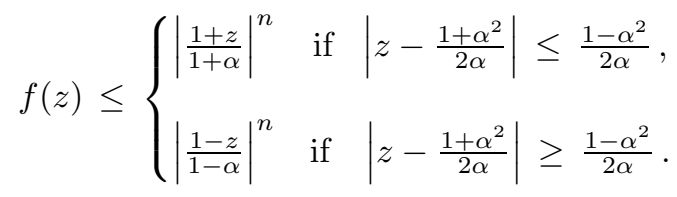

\section{REFERENCES}

1. L.V. Ahlfors, Complex Analysis, 2nd ed., McGraw-Hill Book Company, New York, 1966. MR 32:5844

2. S.N. Bernstein, Sur une propriété des polynômes, Comm. Soc. Math. Kharkow Sér. 214 (1913), pp. 1-6.

3. P. Erdös, On extremal properties of the derivatives of polynomials, Ann. of Math. 41 (1940), pp. 310-313. MR 1:323g

4. P. Erdös, Some remarks on polynomials, Bull. Amer. Math. Soc. 53 (1947), pp. 1169-1176. MR 9:281g

5. I.P. Natanson, Constructive Function Theory, vol. I, Frederick Ungar Publishing Co., Inc., New York, 1964. MR 33:4529a

6. T.J. Rivlin, Chebyshev Polynomials: From Approximation Theory to Algebra and Number Theory, 2nd ed., Wiley, New York, 1990. MR 92a:41016

Department of Mathematics, University of Sofia, James Boucher 5, 1126 Sofia, BulGARIA

Current address: Département de Mathématiques et de Statistique, Université de Montréal, Montréal, Canada H3C 3J7

E-mail address: dryanovd@ere.UMontreal.CA

Département de Mathématiques et de Statistique, Université de Montréal, Montréal, CANADA H3C 3J7

E-mail address: rahmanqi@ere.UMontreal.CA 